\title{
Allocation problems with indivisibilities when preferences are single-peaked
}

\author{
Carmen Herrero • Ricardo Martínez
}

Received: 15 November 2010 / Accepted: 7 February 2011 / Published online: 23 February 2011 (C) The Author(s) 2011. This article is published with open access at SpringerLink.com

\begin{abstract}
We consider allocation problems with indivisible goods when preferences are single-peaked. In this paper we identify the family of efficient, non-manipulable, consistent, and balanced solutions. We refer those solutions as Temporary Satisfaction Methods, that can be viewed as extensions to the indivisible case of the so-called uniform rule.
\end{abstract}

Keywords Allocation problem - Indivisibilities - Single-peaked preferences · Priority standard $\cdot$ Temporary satisfaction methods

JEL Classification D61 $\cdot$ D63 $\cdot$ D74

\begin{abstract}
We are grateful to José Alcalde, François Maniquet, Jordi Massó, Juan D. Moreno-Ternero, William Thomson, Antonio Villar and an anonymous referee for their helpful and stimulating comments. All errors are our sole responsibility. We acknowledge financial support from the Ministerio de Educación y Ciencia and Feder Funds under projects SEJ2007-62656, SEJ2007-67734, ECO2008-03674, Junta de Andalucía under project P06-SEJ-1645 and Grupo PAI SEJ426, and finally from the Instituto Valenciano de Investigaciones Económicas.
\end{abstract}

\section{Herrero $(\varangle)$}

Departamento de Fundamentos del Análisis Económico and Instituto Valenciano de Investigaciones Económicas, University of Alicante \& IVIE, 03080 Alicante, Spain e-mail: carmen.herrero@ua.es

R. Martínez

Departmento de Teoría e Historia Económica, University of Málaga, 03921 Málaga, Spain

e-mail: ricardo_martinez@uma.es 


\section{Introduction}

In this paper we deal with the problem of allocating a fixed amount of indivisible units of an homogeneous good among a group of agents whose preferences are single-peaked. The standard example is that of a group of agents who collectively must supply a certain quantity of labor paid at a fixed rate, in order to complete a task that is a common responsibility, and time comes in indivisible units. Take, for instance, the case of a health center in which there are a certain number of shifts to be covered by doctors, an airline company that has to assign flights to crew members, or a University Department in which some teaching hours should be covered by faculty members. If the agents' (doctors, crew members, professors) disutility of labor is concave, their induced preferences are single-peaked, namely, each individual has an ideal number of units of time to work, and having to work more or less decreases her utility.

The above situation is a particular instance of a general set of problems called allocation problems with indivisibilities when preferences are single-peaked. These problems are described by three elements. First, a set of agents (doctors, crew members, university professors). Second, an amount of indivisible units of time to distribute, called task (number of shifts, flights, or teaching hours). And third, a profile of single-peaked preferences over the number of units of time to work. When the aggregate supply of labor (i.e. the sum of the ideal amounts) differ from the units of time required to complete the task, some procedure should be designed in order to allocate the difference. A rule or solution is a mechanism to distribute the task among the agents according to their preferences.

In the axiomatic method, solutions are justified in terms of the properties they fulfil, and, in general, suitable combinations of desirable properties are used to differentiate among rules. In this paper, we focus on five axioms: efficiency, balancedness (equal agents receive "almost" equal amounts, i.e., they differ at most in one unit), strategy-proofness (it is not in the interest of the agents to misrepresent their preferences), and consistency (stability with respect changes in the set of agents). Balancedness has some "fairness" flavor, whereas strategy-proofness and consistency are "procedural properties".

In this paper we identify the class of rules that, applied to the allotment of indivisible units, are efficient, balanced, strategy-proof, and consistent. Interestingly, any rule in this family can be obtained by a very simple allocation method. In these methods, we start by (temporarily) assigning to each agent her ideal amount of units of time to work, and then, moving away from this temporary assignment on a unit-by-unit basis until achieving the task. We refer to such a family of rules as Temporary Satisfaction methods (TS methods, in short). After (provisionally) assigning each agent her ideal amount, the different rules vary in the way they move away up to reaching the task. We do so by considering types of priority orders, which we call priority standards. They are simply priority orders on agent-number pairs that favor larger numbers. Here, the numbers paired with the agents are interpreted either as agents' peaks or the opposite peaks, depending upon the type of problem in hand (either an excess supply or excess demand problem, respectively).

By far the best-known rule in the continuous case, when the task is perfectly divisible, is the uniform rule, introduced in Benassy (1982), and first characterized in 
Sprumont (1991). It proposes to treat all agents as equally as possible, subject to efficiency. Characterizations of this rule also appear in Ching (1994), Sönmez (1994), Thomson (1994a,b), and Dagan (1996), among others. The uniform rule is efficient, equitable, and non-manipulable. It is worth noting that TS methods can be interpreted as discrete versions of the uniform rule. This statement is supported by two facts. First, the family of TS methods can be characterized by combinations of properties similar to those supporting the uniform rule. And second, for any problem, the allocation prescribed by the uniform rule is the ex-ante expectation of the agents under the application of TS methods, if all plausible priority standards are equally likely.

The rest of the paper is structured as follows: In Sect. 2 we set up the model. In Sect. 3 we analyze the properties our families of rules may fulfil. In Sect. 4 we introduce priority standards, use them to construct TS methods and also present our characterization result. In Sect. 5 we establish the connections between the TS methods and the uniform rule. Finally, in Sect. 6 we conclude with some final remarks. Examples providing the tightness of the characterization are relegated to Appendix A, while proofs are in Appendix B.

\section{Statement of the model}

A preference relation, $R$, defined over $\mathbb{Z}_{+}$is single-peaked if there exists an integer number $p(R) \in \mathbb{Z}_{+}$(called the peak of $R$ ) such that, for each $a, b \in \mathbb{Z}_{+}$,

$$
a P b \Leftrightarrow[(b<a<p(R)) \text { or }(p(R)<a<b)],
$$

where $P$ is the strict preference relation induced by $R$. Let $\mathbb{S}$ denote the class of all single-peaked preferences defined over $\mathbb{Z}_{+}$. Let $\mathbb{N}$ be the set of all potential agents and $\mathcal{N}$ be the family of all finite non-empty subsets of $\mathbb{N}$.

An allocation problem with single-peaked preferences, or simply a problem, is a triple $\boldsymbol{e}=(\boldsymbol{N}, \boldsymbol{T}, \boldsymbol{R})$ in which a fixed number of units $T$ (called task) has to be distributed among a group of agents, $N \in \mathcal{N}$, whose preferences over consumption are single-peaked, $R=\left(R_{i}\right)_{i \in N} \in \mathbb{S}^{N}$. Let $\mathbb{A}$ denote the class of problems.

For each problem, we face the question of finding a division of the task among the agents. An allocation for $e \in \mathbb{A}$ is a list of integers, $x \in \mathbb{Z}_{+}^{N}$, satisfying the condition of being a complete distribution of the task, i.e., $\sum_{i \in N} x_{i}=T$. Let $\boldsymbol{X}(\boldsymbol{e})$ be the set of all allocations for $e \in \mathbb{A}$. A rule is a function, $\boldsymbol{F}: \mathbb{A} \longrightarrow \bigcup_{e \in \mathbb{A}} X(e)$, that selects, for each problem $e \in \mathbb{A}$, a single allocation $F(e) \in X(e)$.

\section{Properties}

Our goal in this paper is to obtain efficient, strategy-proof, consistent, and balanced rules to solve allocation problems with indivisibilities when preferences are singlepeaked.

A basic requirement a rule must satisfy is efficiency. An allocation is efficient if there is no other allocation in which all the agents are better-off. Efficiency requires that the rule selects efficient allocations. 
Efficiency. For each $e \in \mathbb{A}$, there is no allocation $x \in X(e)$ such that, for each $i \in N, x_{i} R_{i} F_{i}(e)$, and for some $j \in N, x_{j} P_{j} F_{j}(e)$.

As Sprumont (1991) points out, the principle of efficiency is equivalent to asking each agent to provide no more than her peak when the aggregate supply exceeds the task, and no less than her peak otherwise. That is, if $F$ is efficient, $F_{i}(e) \leq p\left(R_{i}\right)$ for each $i \in N$ if $\sum_{i \in N} p\left(R_{i}\right) \geq T$, and $F_{i}(e) \geq p\left(R_{i}\right)$ for each $i \in N$ if $\sum_{i \in N} p\left(R_{i}\right) \leq T$.

Normally, agents report their preferences directly to a central planner. This opens the door to the possibility of manipulation from those agents. Strategy-proofness requires that it is not in the benefit of any agent to misrepresent her preference. ${ }^{1}$

Strategy-proofness: For each $e=\left(N, T,\left(R_{i}, R_{-i}\right)\right) \in \mathbb{A}$ and each $e^{\prime}=(N, T$, $\left.\left(R_{i}^{\prime}, R_{-i}\right)\right) \in \mathbb{A}, F_{i}(e) R_{i} F_{i}\left(e^{\prime}\right)$.

Now, we deal with a sort of robustness condition with respect to changes in population. Suppose that, after solving the problem $e=(N, T, R) \in \mathbb{A}$, a proper subset of agents, $S \subset N$, decides to reallocate their total share of the task, that is, they face a new allocation problem: $\left(S, \sum_{i \in S} x_{i}, R_{S}\right)$, where $R_{S}=\left(R_{i}\right)_{i \in S}$ and $x$ is the allocation corresponding to apply the rule to the problem $e$. Consistency requires that each agent $i \in S$ receives the same amount of units in problem $\left(S, \sum_{i \in S} x_{i}, R_{S}\right)$ as she did in problem $e$. In other words, the new reallocation is only a restriction to the subset $S$ of the initial one. ${ }^{2}$

Consistency: For each $e \in \mathbb{A}$, each $S \subset N$, and each $i \in S, F_{i}(e)=F_{i}\left(S, \sum_{j \in S}\right.$ $\left.F_{j}(e), R_{S}\right)$.

There are different traditional fairness properties: one is to treat identical agents identically. Together with efficiency, it simply means that agents with identical preferences should receive the same amount. This requirement cannot be unconditionally met in the indivisibilities case. We relax this property by simply asking identical agents to be "almost" indifferent among their allotted shares of the task. More precisely, equal agents get amounts that can differ only in one unit, which represents the size of the indivisibility. ${ }^{3}$

Balancedness. For each $e \in \mathbb{A}$ and each $\{i, j\} \subseteq N$, if $R_{i}=R_{j}$ then $\mid F_{i}(e)-$ $F_{j}(e) \mid \leq 1$

Notice that balancedness does not require to treat identical agents identically, even when this is possible. Consider, for instance, the problem $e \in \mathbb{A}$ where $N=\{1,2,3\}$, $T=5$, and $R$ is such that $R_{1}=R_{2}$ and $p(R)=(3,3,2)$. A balanced rule may select the allocation $F(e)=(1,2,2)$. However, in this problem it is possible to treat equally agents 1 and 2 by choosing the allocation $(2,2,1)$. Why then not to impose an axiom that requires to treat identical agents identically when it is possible? Essentially because it is incompatible with strategy-proofness. Indeed, let us

\footnotetext{
1 The notation $R_{-i}$ refers to the preference profile $R$ where agent $i$ has been deleted, i.e., $R_{-i}=R_{N \backslash\{i\}}$.

2 The concept of consistency in the divisible case is introduced in Thomson (1994b). See also Thomson (1998b) for a widely exposition of consistency and its converse.

3 Young (1994) and Herrero and Martínez (2008) formulate a similar property in the claims problems context.
} 
consider a profile $R \in \mathbb{S}^{N}$ such that $p\left(R_{i}\right)=2$ for all $i \in N$. Without loss of generality we can assume that $F(N, 1, R)=(1,0, \ldots, 0)$. Now, if one agent $i \in N-1$ changes her preference to $R_{i}^{\prime}$ such that $p\left(R_{i}^{\prime}\right)=4$, in application of equal treatment when possible, it must happen that $F_{j}\left(N, 1,\left(R_{1}, R_{i}^{\prime}, R_{-1 i}\right)\right)=0$ for all $j \neq i$ and $F_{i}\left(N, 1,\left(R_{1}, R_{i}^{\prime}, R_{-1 i}\right)\right)=1>0=F_{i}\left(N, 1,\left(R_{1}, R_{i}, R_{-1 i}\right)\right)$, which contradicts strategy-proofness.

\section{A family of rules. Temporary satisfaction methods}

Now we face the question of finding rules satisfying the properties formulated in Sect. 3. To do so, we shall consider a two-step procedure.

A priority standard is a linear order over the cartesian product $\mathbb{N} \times \mathbb{Z}$ (potential agent/number), such that pairs involving larger numbers go first. ${ }^{4}$

Priority standard, $\sigma$. It is a linear ordering on the set of all pairs $(i, a) \in \mathbb{N} \times \mathbb{Z}$ such that, for all $i, j \in \mathbb{N}$ and all $a \in \mathbb{Z},(i, a+1) \sigma(j, a)$. Let $\Sigma$ denote the class of all priority standards.

Let $\left\{\left(i, a_{i}\right)\right\}_{i \in L}$ be a collection of pairs agent-number. Let $\sigma$ be a priority standard. The pair with the highest priority in $\left\{\left(i, a_{i}\right)\right\}_{i \in L}$ according to $\sigma$ is the pair $\left(i, a_{i}\right)$ such that $\left(i, a_{i}\right) \sigma\left(j, a_{j}\right)$ for all $j \in L \backslash\{i\}$.

Priority standards will be used to accommodate all units of either excess demand or excess supply on a unit-by-unit basis, after giving (temporarily) all agents their peaks.

For any given problem $e$, and for each agent in $e$, we consider all pairs involving that agent together with any number smaller or equal than her peak (whenever we are in an excess-supply problem), or the opposite of her peak otherwise. The priority standard orders all those pairs. Then, we start by allocating each agent her peak. Now, we move away from the peaks on a unit by unit basis, according to the priority standard up to achieving the task. Different priority standards give rise to different ways of solving problems, i.e., different rules. We call those rules Temporary Satisfaction methods (in short, TS methods).

Temporary satisfaction method associated to $\sigma, \boldsymbol{T} \boldsymbol{S}^{\sigma}$ : Let $e \in \mathbb{A}$. Start by giving each agent her peak. Now we distinguish two cases.

1. If we face an excess-supply problem, i.e., $\sum_{i \in N} p\left(R_{i}\right) \geq T$. In this case we have to remove some units from the temporary allocation. Take the collection of all pairs $\left\{\left(i, a_{i}\right)\right\}_{i \in N}$ with $a_{i} \leq p\left(R_{i}\right)$. Those pairs are ranked according to $\sigma$. Identify the pair with the highest priority, subtract one unit from the temporary assignment of this agent, and remove the pair from the collection. Identify the pair with the next highest priority according to $\sigma$, and proceed in the same way until achieving the task.

2. If we face an excess-demand problem, i.e., $\sum_{i \in N} p\left(R_{i}\right) \leq T$. In this case we have to allocate some extra units, $T^{\prime}=T-\sum_{i \in N} p\left(R_{i}\right)$. We shall proceed in the following way. Take the collection of all pairs $\left\{\left(i, a_{i}\right)\right\}_{i \in N}$ with $a_{i} \leq-p\left(R_{i}\right)$. Those pairs are ranked according to $\sigma$. Identify the pair with the highest priority, give one unit of the remaining task $T^{\prime}$ to this agent, and remove the pair from

$\overline{4}$ Related notions appear in Young (1994) and Herrero and Martínez (2008). 
the collection. Identify the pair with the next highest priority according to $\sigma$, and proceed in the same way until $T^{\prime}$ runs out.

The next example illustrates how temporary satisfaction methods work.

Example 4.1 Let $N=\{1,2,3\}$, and let $\sigma$ be a priority standard such that $(1, x) \sigma(2, x) \sigma(3, x)$ if $x$ is odd, and $(1, x) \sigma(3, x) \sigma(2, x)$ if $x$ is even. Now, consider the allocation problem where $N=\{1,2,3\}, T=6$, and $R=\left(R_{1}, R_{2}, R_{3}\right)$ such that $R_{2}=R_{3}$ and $p(R)=(1,5,5)$. We start by temporarily fully satisfying all the agents. This means giving 11 units while we only have 6 , that is, we need to subtract 5 . To do that, we then take all the pairs $\{(1,1),(1,0),(1,-1),(1,-2), \ldots,(2,5),(2,4)$, $(2,3), \ldots,(3,5),(3,4),(3,3), \ldots\}$. According to $\sigma$, the pair with the highest priority is $(2,5)$. Then we subtract one unit from agent 2 . The pair with the next highest priority is $(3,5)$. Then we subtract the second unit from agent 3 , and so on. Applying the aforementioned procedure, we obtain the result that $T S^{\sigma}(e)=(1,2,3)$. The table below presents the procedure.

$$
\begin{aligned}
& \text { highest priority pair } \quad(2,5) \quad(3,5) \quad(3,4) \quad(2,4) \quad(2,3) \\
& \text { assignment } \quad(1,5,5)(1,4,5)(1,4,4)(1,4,3)(1,3,3)(\mathbf{1}, \mathbf{2}, \mathbf{3})
\end{aligned}
$$

If the number of units to allot is $T=14$, then $T S^{\sigma}(e)=(4,5,5)$. The table below details the functioning of the procedure.

$$
\begin{aligned}
& \text { highest priority pair } \quad(1,-1)(1,-2)(1,-3) \\
& \text { assignment } \quad(1,5,5)(2,5,5)(3,5,5)(\mathbf{4}, \mathbf{5}, \mathbf{5})
\end{aligned}
$$

As mentioned above, our goal is to identify efficient, balanced, strategy-proof, and consistent rules. In our main result, we obtain that there is a family of rules compatible with these four requirements, the family of TS methods. The proof, preceded by some lemmas, is relegated to Appendix B.

Theorem 4.1 A rule $F$ satisfies efficiency, balancedness, strategy-proofness, and consistency if and only if there exists a priority standard $\sigma \in \Sigma$ such that $F=T S^{\sigma}$.

Moulin (1999) identifies the family of rules that are efficient, resource monotonic (an increase in the task does not result in the decrease of some agent's assignment), strategy-proof, and efficient. Those solutions are the so called fixed path rules. In the previous theorem we substitute resource monotonicity by balancedness, and we end up with the temporary satisfaction methods. Our TS methods also satisfy resource monotonicity, and therefore are particular cases of the fixed path rules when, using the terminology in Moulin (1999), the priority steps do not draw away from equitability by more than one unit.

\section{Relations between the discrete and the continuum}

In the previous section we obtained a characterization for the family of TS methods. The properties used in such a result (Theorem 4.1) are very much related to those used 
by Ching (1994) to characterize the uniform rule. ${ }^{5}$ This fact suggests the existence of a close relationship between our TS methods and the uniform rule. Actually, TS methods can be interpreted as discrete versions of the uniform rule in the following sense. The allocation prescribed by the uniform rule is the ex-ante expectation of the agents under the application of TS methods, if all priority standards are equally likely.

Proposition 5.1 Let $e \in \mathbb{A}$. Let $\Sigma_{e}$ denote the subset of $\Sigma$ of the different priority standards involved in problem e. ${ }^{6}$ Then

$$
\frac{1}{\left|\Sigma_{e}\right|} \sum_{\sigma \in \Sigma_{e}} T S^{\sigma}(e)=u(e)
$$

An additional point of similarity between the uniform rule and the TS-methods can be obtained by defining these methods in an alternative way, that reminds of the definition of the continuous uniform rule, with one proviso: the impossibility of finding a single "uniform" value $\lambda$, for any problem, and instead, moving this common number between two subsequent integers:

Let $\sigma \in \Sigma$ be a priority standard. Let $e=(N, T, R) \in \mathbb{A}$ be a problem. Let $\lambda(e) \in \mathbb{Z}_{+}$be the amount such that

$$
\begin{gathered}
\sum_{i \in N} \min \left\{p\left(R_{i}\right), \lambda(e)-1\right\}<T \leq \sum_{i \in N} \min \left\{p\left(R_{i}\right), \lambda(e)\right\} \quad \text { if } \pi(e) \geq T \\
\sum_{i \in N} \max \left\{p\left(R_{i}\right), \lambda(e)\right\} \leq T<\sum_{i \in N} \max \left\{p\left(R_{i}\right), \lambda(e)+1\right\} \quad \text { if } \pi(e)<T,
\end{gathered}
$$

where $\pi(e)=\sum_{i \in N} p\left(R_{i}\right)$. Now, let us define $N(e)=\left\{i \in N: p\left(R_{i}\right) \geq \lambda(e)\right\}$ and $T^{\prime}(e)=\sum_{i \in N} \min \left\{p\left(R_{i}\right), \lambda(e)\right\}-T$ if $\pi(e) \geq T$, and $N(e)=\left\{i \in N: p\left(R_{i}\right) \leq\right.$ $\lambda(e)\}$ and $T^{\prime}(e)=T-\sum_{i \in N} \max \left\{p\left(R_{i}\right), \lambda(e)\right\}$ if $\pi(e)<T$. Let $f: \mathbb{A} \longrightarrow 2^{\mathbb{N}}$ be a function such that, for each $e \in \mathbb{A}, f(e) \subset N(e)$ and $|f(e)|=T^{\prime}(e)$. Each function $f$ selects the agents in $N(e)$ that receives one of the units in $T^{\prime}(e)$.

For each $M \in \mathcal{N}$, let $\mathbf{1}_{M}$ be an $m$-vector in $\{0,1\}^{M}$ such that

$$
\left(\mathbf{1}_{M}\right)_{i}= \begin{cases}1 & \text { if } i \in M \\ 0 & \text { otherwise }\end{cases}
$$

\footnotetext{
5 Under the assumption that the task is completely divisible, one of the most widely studied rules is the so-called uniform rule. The idea underlying this solution is equality distribution, subject to efficiency, of the task.

Uniform rule, $u$ : For each $e \in \mathbb{A}$, selects the unique vector $u(e) \in \mathbb{R}^{N}$ such that: If $\sum_{i \in N} p\left(R_{i}\right) \geq T$, then $u(e)=\min \left\{p\left(R_{i}\right), \lambda\right\}$ for some $\lambda \in \mathbb{R}$ such that $\sum_{i \in N} \min \left\{p\left(R_{i}\right), \lambda\right\}=T$. And, if $\sum_{i \in N} p\left(R_{i}\right) \leq T$, then $u(e)=\max \left\{p\left(R_{i}\right), \lambda\right\}$ for some $\lambda \in \mathbb{R}$ such that $\sum_{i \in N} \max \left\{p\left(R_{i}\right), \lambda\right\}=T$.

6 In $\Sigma$ we consider all priority standards over $\mathbb{N} \times \mathbb{Z}$. Notice that, for a given $e$, not all of them rank the pairs $\left(i, a_{i}\right)$ involved in that particular problem in different ways. $\Sigma_{e}$ denotes precisely the subset of those different standards.
} 
We can define the temporary satisfaction method associated to priority standard $\sigma \in \Sigma$ as follows ${ }^{7}$

$$
T S_{i}^{\sigma}(e)=\left\{\begin{array}{l}
\min \left\{p\left(R_{i}\right), \lambda(e)\right\}-\left(\mathbf{1}_{f(e)}\right)_{i} \text { where }[N(e) \backslash f(e), \lambda(e)] \sigma[f(e), \lambda(e)] \text { if } \pi(e) \geq T \\
\max \left\{p\left(R_{i}\right), \lambda(e)\right\}+\left(\mathbf{1}_{f(e)}\right)_{i} \text { where }[f(e), \lambda(e)] \sigma[N(e) \backslash f(e), \lambda(e)] \text { if } \pi(e)<T
\end{array}\right.
$$

\section{Final remarks}

In this work we have considered allocation problems with indivisible goods when preferences are single-peaked, that is, problems in which the task, the allocations and the preferences are only defined over the set of integer numbers. Our goal is to obtain rules that represent a good trade-off between efficiency, stability (non-manipulability and consistency), and equity (balancedness). To do that, we have defined the Temporary Satisfaction methods. These methods are the only efficient, strategy-proof, consistent and balanced rules.

In Barberà et al. (1997), a family of allocation rules for the division problem with single-peaked preferences in the continuous case are introduced. The so called sequential allotment rules (SAR in short) are the only ones satisfying efficiency, strategyproofness, and replacement monotonicity, a property that states that if a change in one agent's preferences results in that individual receiving a larger (resp. smaller) share of the task as before, then all other individuals' allotments are no larger (resp. no smaller) than before. ${ }^{8}$ The construction of SARs can be adapted to the indivisibilities case, with one proviso: unlike the continuous case, here the guaranteed levels depend on the set of agents and the task. Then, for a fixed number of agents, the family of SARs in the case of indivisibilities also satisfy efficiency, strategy-proofness and replacement monotonicity. It is, nonetheless, an open question whether those are the only rules in that case fulfilling these requirements. Also, and for any fixed number of agents, any $T S^{\sigma}$ rule can be obtained by a SAR rule, meaning that in the fixed agent case, $T S$ rules are a subset of the family of sequential allotment rules.

It is easy to adapt the definition of SAR in the indivisibilities case to get rules that also fulfill balancedness. Nonetheless, for the fixed agents case, not all SAR balanced rules belong to the family of $T S$ methods. This means that, as it happens in our case, consistency seems to be crucial. Nonetheless, there is still an open question how to obtain consistent SARs rules, both in the discrete and continuous framework.

Our procedure and the one proposed in Barberà et al. (1997) are both sequential, but they are significantly different. While our procedure starts, for any problem, by assigning each agent her peak, and then moving away on a unit-by-unit basis, their procedure starts in a feasible (non efficient) allocation, and then adjusting some agents to their peaks, and distributing the remaining amounts following an unspecified function. It is far from clear which conditions should be imposed on such adjustment function to comply with consistency.

\footnotetext{
7 For each pair of agents sets $M, M^{\prime} \subseteq N$ such that $M \cap M^{\prime}=\phi$, and each integer $a \in \mathbb{Z}_{+}$, we write $[M, a] \sigma\left[M^{\prime}, a\right]$ when $(i, a) \sigma(j, a)$ for all $i \in M$ and all $j \in M^{\prime}$.

8 Replacement monotonicity is also satisfied by the $T S$ methods.
} 
We have also noticed that, our family of solutions can be characterized by combinations of properties similar to those supporting the characterizations of the uniform rule. Moreover, the allocation selected by the uniform rule can be interpreted as the expected allocation of the Temporary Satisfaction methods, if all plausible priority standards are equally likely.

In comparing Theorem 4.1 with the characterization results in Ching (1994) and Sprumont (1991) for the continuous case, we observe two differences. First, anonymity cannot be imposed in the presence of indivisibilities. Here, the equity requirement is captured by balancedness, a less demanding property that does not guarantee equal treatment, even when this is feasible. And, secondly, unlike Ching (1994) and Sprumont (1991), consistency plays an important and inescapable role. Interestingly, we have an additional property to that in Ching (1994), namely, consistency. By means of that additional property we characterize a full family of solutions in the indivisibilities case, whereas Ching (1994) characterizes a single rule (the uniform rule) in the perfectly divisible case.

A problem closely related with the one studied here is the case of allocation problems with indivisibilities when preferences are single-plateaud. If we assume that preferences are single-peaked on the continuum, and then think of the indivisibilities case as the restriction of those preferences to that case, then preferences on the indivisible case could be single-plateaud instead of single-peaked. Nonetheless, when agents are aware of the indivisibilities, single-peakedness is as likely to appear as in the continuous case. The case in which preferences are single-plateaud in this context is left for future research.

\section{Appendix A. On the tightness of characterization result}

We now present a collection of examples to illustrate the independence of properties used in Theorem 4.1.

Example 6.1 Let $\succ: \mathbb{N} \longrightarrow \mathbb{Z}_{++}$be an order defined over the set of potential agents such that agent labeled $i$ has priority over agent labeled $i+1$, i.e., $i \succ i+1$. The rule $G^{\succ}$ works as follows. Let $e \in \mathbb{A}$. Give to each agent the integer part of the equal split allocation, ${ }^{9}$ that is $\left\lfloor\frac{T}{n}\right\rfloor$ for each $i \in N$. If no unit remains we have finished. If some units, $T^{\prime}=T-n \cdot\left\lfloor\frac{T}{n}\right\rfloor$, remain, then allot one unit to the $T^{\prime}$ agents with the highest priority according to $\succ$.

Example 6.2 Consider a serial dictator rule with a fixed order, $\succ$, on the set of agents, $S D^{\succ}$.

Example 6.3 Alternatively to the Temporary Satisfaction methods, in which we start by fully satisfying all the agents, we may decide to allocate the task unit by unit, starting by giving nothing to each agent. Let $\sigma \in \Sigma$. Then

Up method associated to $\sigma, \boldsymbol{U}^{\sigma}$ : Let $e \in \mathbb{A}$. For each agent in $e$, consider her preferred labor supply in $e$, that is, her peak $p\left(R_{i}\right)$, and take all pairs $\left\{\left(i, a_{i}\right)\right\}_{i \in N}$ with

9 We denote by $\lfloor a\rfloor$ the smallest integer number that is no greater than $a$. 
Table 1 Independence of properties

\begin{tabular}{lllll}
\hline Property & $G^{\succ}$ & $S D^{\succ}$ & $U^{\sigma}$ & $F^{\left(\sigma_{1}, \sigma_{2}\right)}$ \\
\hline Efficiency & $\mathrm{N}$ & $\mathrm{Y}$ & $\mathrm{Y}$ & $\mathrm{Y}$ \\
Balancedness & $\mathrm{Y}$ & $\mathrm{N}$ & $\mathrm{Y}$ & $\mathrm{Y}$ \\
Strategy-proofness & $\mathrm{Y}$ & $\mathrm{Y}$ & $\mathrm{N}$ & $\mathrm{Y}$ \\
Consistency & $\mathrm{Y}$ & $\mathrm{Y}$ & $\mathrm{Y}$ & $\mathrm{N}$ \\
\hline
\end{tabular}

$a_{i} \leq p\left(R_{i}\right)$. Identify the pair with the highest priority, according to $\sigma$, and assign the first unit of the task to the agent in that pair from $\left\{\left(i, a_{i}\right)\right\}_{i \in N}$. Remove the pair. Identify the agent in the pair with the next highest priority, and assign this agent the second unit of the task. Proceed in the same way until the task is exhausted.

Example 6.4 This rule, $F^{\left(\sigma_{1}, \sigma_{2}\right)}$, can be defined as follows. Let $\sigma_{1}, \sigma_{2} \in \Sigma$ be two different priority standards such that $(i, x) \sigma_{1}(i+1, x)$ and $(i+1, x) \sigma_{2}(i, x)$. Then, we define the solution $F^{\left(\sigma_{1}, \sigma_{2}\right)}$ as

$$
F^{\left(\sigma_{1}, \sigma_{2}\right)}(e)= \begin{cases}T S^{\sigma_{1}}(e) & \text { if }|N|=2 \\ T S^{\sigma_{2}}(e) & \text { otherwise }\end{cases}
$$

Table 1 shows the independence of properties in Theorem 4.1.

\section{Appendix B. Proofs of the results}

We present in this appendix the proof of Theorem 4.1 and Proposition 5.1, preceded by some technical results.

Let us consider an allocation for a problem with the following feature. For each two-agent subset, the rule chooses the restriction of that allocation for the associated reduced problem to this agent subset. Converse consistency requires the allocation to be the one selected by the rule for the original problem. This property was formulated by Chun (1999) in the context of claims problems.

Let $c . \operatorname{con}(e ; F) \equiv\left\{x \in \mathbb{Z}_{+}^{N}: \sum_{i \in N} x_{i}=T\right.$ and for all $S \subset N$ such that $|S|=$ $\left.2, x_{S}=F\left(S, \sum_{i \in S} x_{i}, R_{S}\right)\right\}$

Converse consistency. For each $e \in \mathbb{A}, c \cdot \operatorname{con}(e ; F) \neq \phi$, and if $x \in c \cdot \operatorname{con}(e ; F)$, then $x=F(e)$.

Peak-only says that an agent's allocation depends only on his preferred consumption.

Peak-only. For each $e=\left(N, T,\left(R_{i}, R_{-i}\right)\right) \in \mathbb{A}$ and each $e^{\prime}=\left(N, T,\left(R_{i}^{\prime}, R_{-i}\right)\right) \in$ $\mathbb{A}$ such that $p\left(R_{i}^{\prime}\right)=p\left(R_{i}\right)$, then $F_{i}(e)=F_{i}\left(e^{\prime}\right)$.

One-sided resource monotonicity, considers the case in which there is a change in the task that does not alter the type of rationing associated to the initial problem, i.e, if initially we have to ration labor, it is still labor to be rationed after the task has increased or, otherwise, if in the initial problem we have to ration leisure, then again, we have excess of labor to allocate even after the decreasing of the task. In either case, the property states that no agent should suffer. 
One-sided resource monotonicity: For each $e, e^{\prime} \in \mathbb{A}$ such that $e=(N, T, R)$ and $e^{\prime}=\left(N, T^{\prime}, R\right)$. If (a) $\sum_{j \in N} p\left(R_{j}\right) \geq T^{\prime}>T$, or (b) $\sum_{j \in N} p\left(R_{j}\right) \leq T^{\prime}<T$. Then for each $i \in N, F_{i}\left(e^{\prime}\right) R_{i} F_{i}(e)$.

Lemma 6.1 (Elevator Lemma, (Thomson (1998a))) If a rule $F$ is consistent and coincides with a conversely consistent rule $F^{\prime}$ in the two agent case, then it coincides with $F^{\prime}$ in general.

The following Lemma is due to Ching (1994), Even though the author proves the result for the continuous case, it is still valid in presence of indivisibilities.

Lemma 6.2 Efficiency and strategy-proofness together imply peak-only.

Lemma 6.3 Efficiency, one-sided resource monotonicity, and consistency together imply converse consistency.

Proof Let $e \in \mathbb{A}$. By consistency the set $\operatorname{c.con}(e ; F) \neq \phi$. Let $x, y \in \operatorname{cocon}(e ; F)$ with $x \neq y$. We distinguish two cases.

Case 1. If $\sum_{i \in N} p\left(R_{i}\right) \geq T$. Since, $x \neq y$, there exists $k \in N$ such that $x_{k}>y_{k}$. Consider each two-agent set $S=\{k, j\}$ with $j \in N$ and $j \neq k$. Since $x, y \in \operatorname{cocon}(e ; F), x_{S}=F\left(S, x_{j}+x_{k}, R_{S}\right)$ and $y_{S}=F\left(S, y_{j}+y_{k}, R_{S}\right)$. By efficiency and one-sided resource monotonicity, $x_{j} \geq y_{j}$. This fact, together with $x_{k}>y_{k}$, and $\sum_{i \in N} x_{i}=T=\sum_{i \in N} y_{i}$ yields a contradiction.

Case 2. If $\sum_{i \in N} p\left(R_{i}\right) \leq T$. There exists $k \in N$ such that $x_{k}<y_{k}$. Consider each two-agent set $S=\{k, j\}$ with $j \in N$ and $j \neq k$. Since $x, y \in$ $\operatorname{c.con}(T, R ; F), x_{S}=F\left(S, x_{j}+x_{k}, R_{S}\right)$ and $y_{S}=F\left(S, y_{j}+y_{k}, R_{S}\right)$. By efficiency and one-sided resource monotonicity, $x_{j} \leq y_{j}$. This fact, together with $x_{k}<y_{k}$, and $\sum_{i \in N} x_{i}=T=\sum_{i \in N} y_{i}$ yields a contradiction.

Lemma 6.4 Let $F$ be a rule satisfying efficiency, balancedness, and strategy-proofnes. Let $e, e^{\prime} \in \mathbb{A}$ be two problems involving two agents, $\{i, j\}, e=(\{i, j\}, T,(R, R)) ; e^{\prime}=$ $\left(\{i, j\}, T,\left(R^{\prime}, R^{\prime}\right)\right)$ such that either both $2 p(R), 2 p\left(R^{\prime}\right)$ are strictly larger or both strictly smaller than $T$. Then, $F(e)=F\left(e^{\prime}\right)$.

Proof First, consider the case where $2 p(R)>T, 2 p\left(R^{\prime}\right)>T$. Let $R^{\prime \prime}$ be such that $p\left(R^{\prime \prime}\right)=\frac{T+1}{2}$, and let $e^{\prime \prime}=\left(\{i, j\}, T,\left(R^{\prime \prime}, R^{\prime \prime}\right)\right)$. We shall prove that $F(e)=$ $F\left(e^{\prime \prime}\right)=F\left(e^{\prime}\right)$.

If $T$ is even, balancedness implies the result. Let $T=2 \lambda+1$, for some $\lambda \in \mathbb{Z}$, and suppose, w.l.o.g., that $F\left(e^{\prime \prime}\right)=(\lambda, \lambda+1)$, whereas $F(e)=(\lambda+1, \lambda)$. This is the only possibility of discrepancy because of efficiency and balancedness. Since $p(R) \geq p\left(R^{\prime \prime}\right)=\lambda+1$, agent $j$ is happier in problem $e^{\prime \prime}$ than he is in problem $e$, and it is the other way around for agent $i$. Additionally, strategy-proofness implies that

$$
F_{i}\left(\{i, j\}, T,\left(R, R^{\prime \prime}\right)\right) \leq \lambda ; \quad F_{j}\left(\{i, j\}, T,\left(R, R^{\prime \prime}\right)\right) \leq \lambda
$$

The first inequality follows from agent $i$ 's inability to get a better result when misrepresenting his preferences in problem $e^{\prime \prime}$, while the second inequality follows from 
agent $j$ 's inability to benefit from misrepresenting his preferences in problem $e$. But, if this is the case,

$$
F_{i}\left(\{i, j\}, T,\left(R, R^{\prime \prime}\right)\right)+F_{j}\left(\{i, j\}, T,\left(R, R^{\prime \prime}\right)\right) \leq 2 \lambda<T
$$

which is a contradiction with $F$ being a rule.

The case where $2 p(R)<T, 2 p\left(R^{\prime}\right)<T$ is analogous.

Proof of Theorem 4.1 It is easy to check that each $T S^{\sigma}$ satisfies the four properties. Conversely, let $F$ be a rule satisfying all the properties. We divide the rest of the proof into two steps.

Step 1. Definition of the priority standard. Let us define the order $\sigma \in \Sigma$ as follows

$$
\begin{aligned}
a>b & \Rightarrow(i, a) \sigma(j, b) \\
a=b & \Rightarrow\left[(i, a) \sigma(j, b) \Leftrightarrow F_{i}\left(\{i, j\}, 2 a-1,\left(R_{i}, R_{j}\right)\right)=a-1\right],
\end{aligned}
$$

where $R_{i}$ and $R_{j}$ are two single-peaked preference relations such that $p\left(R_{i}\right)=a=b=p\left(R_{j}\right)$ (by Lemma 6.2 it is enough to consider the peaks). It is straightforward to see that such a $\sigma$ is complete and antisymmetric. Let us show that $\sigma$ is transitive. Suppose that there exist $\{i, j, k\} \subseteq \mathbb{N}$ such that $(i, x) \sigma(j, y),(j, y) \sigma(k, z)$, but $(k, z) \sigma(i, x)$. By construction and peak-only (implied by efficiency and strategy-proofness according to Lemma 6.2), this can only happen when $x=y=z$. By the definition of $\sigma$, in such a case, $F_{i}\left(\{i, j\}, 2 x-1,\left(R_{i}, R_{j}\right)\right)=x-1, F_{j}\left(\{j, k\}, 2 x-1,\left(R_{j}, R_{k}\right)\right)=x-1$, and $F_{k}\left(\{k, i\}, 2 x-1,\left(R_{k}, R_{i}\right)\right)=x-1$, where $p\left(R_{i}\right)=p\left(R_{j}\right)=p\left(R_{k}\right)=$ $x=y=z$. Consider the problem $\left(\{i, j, k\}, 3 x-2,\left(R_{i}, R_{j}, R_{k}\right)\right)$. There are only three possible allocations: $(x-1, x-1, x),(x-1, x, x-1)$, and $(x, x-1, x-1)$. Suppose that $F\left(\{i, j, k\}, 3 x-2,\left(R_{i}, R_{j}, R_{k}\right)\right)=(x-1, x-$ $1, x)$, by consistency, $F_{k}\left(\{i, k\}, 2 x-1,\left(R_{i}, R_{k}\right)\right)=x$, achieving in this way a contradiction with $F_{k}\left(\{i, k\}, 2 x-1,\left(R_{i}, R_{j}\right)\right)=x-1$. An analogous argument is applied if $F\left(\{i, j, k\}, 3 x-2,\left(R_{i}, R_{j}, R_{k}\right)\right)=(x-1, x, x-1)$, or if $F\left(\{i, j, k\}, 3 x-2,\left(R_{i}, R_{j}, R_{k}\right)\right)=(x, x-1, x-1)$. Therefore $(i, x) \sigma(k, z)$, and then $\sigma$ is transitive.

Step 2. Let us now prove that $F=T S^{\sigma}$. It is straightforward that $T S^{\sigma}$ is efficient, one-sided resource monotonic, and consistent, then, by Lemma 6.3, $T S^{\sigma}$ is conversely consistent. Therefore, in application of Lemma 6.1, it is enough to show that $F=T S^{\sigma}$ in the two-agent case. Then, let us consider the problem $e=(S, T, R) \in \mathbb{A}$ where $S=\{i, j\}$. Without loss of generality we can assume that $p\left(R_{i}\right) \leq p\left(R_{j}\right)$. We analyze the case in which $p\left(R_{i}\right)+p\left(R_{j}\right) \geq T$. The other case is completely analogous. We distinguish the following cases:

Case 1. If $R_{i}=R_{j}$ and $T$ is even. By balancedness, $F(e)=\left(\frac{T}{2}, \frac{T}{2}\right)=$ $T S^{\sigma}(e)$.

Case 2. If $R_{i}=R_{j}$ and $T$ is odd. If $T=2 p\left(R_{i}\right)-1$, by the definition of the priority standard, $F(e)=T S^{\sigma}(e)$. If $T<2 p\left(R_{i}\right)-1$, 
by Lemma $6.4, F(e)=F\left(S, T,\left(R_{i}^{\prime}, R_{j}^{\prime}\right)\right)$, where $R_{i}^{\prime}=R_{j}^{\prime}$ and $p\left(R_{i}^{\prime}\right)=p\left(R_{j}^{\prime}\right)=\frac{T+1}{2}$. And then, $F(e)=F\left(S, T,\left(R_{i}^{\prime}, R_{j}^{\prime}\right)\right)=$ $T S^{\sigma}\left(S, T,\left(R_{i}^{\prime}, R_{j}^{\prime}\right)\right)=T^{\sigma}(e)$.

Case 3. If $F_{i}(e) \leq F_{j}(e) \leq p\left(R_{i}\right) \leq p\left(R_{j}\right)$. By efficiency and strategyproofness, $F_{i}(e)=F_{i}\left(S, T,\left(R_{j}, R_{j}\right)\right)=T S_{i}^{\sigma}\left(S, T,\left(R_{j}, R_{j}\right)\right)=$ $T S_{i}^{\sigma}(e)$. And then $F(e)=T S^{\sigma}(e)$.

Case 4. If $F_{j}(e) \leq F_{i}(e) \leq p\left(R_{i}\right) \leq p\left(R_{j}\right)$. By efficiency and strategyproofness, $F_{j}(e)=F_{j}\left(S, T,\left(R_{i}, R_{i}\right)\right)=T S_{j}^{\sigma}\left(S, T,\left(R_{i}, R_{i}\right)\right)=$ $T S_{j}^{\sigma}(e)$. And then $F(e)=T S^{\sigma}(e)$.

Case 5. If $F_{i}(e) \leq p\left(R_{i}\right)<F_{j}(e) \leq p\left(R_{j}\right)$. By efficiency and strategy-proof, $F_{i}(e)=F_{i}\left(S, T,\left(R_{j}, R_{j}\right)\right)=T S_{i}^{\sigma}\left(S, T,\left(R_{j}, R_{j}\right)\right)=$ $T S_{i}^{\sigma}(e)$. And then $F(e)=T S^{\sigma}(e)$.

Then, $F$ coincides with $T S^{\sigma}$ in the two-agent case and, therefore, they do so in general.

Proof of Proposition 5.1 Let $e \in \mathbb{A}$ be a problem. Let $p=\left(p_{1}, \ldots, p_{n}\right)$ be the vector of peaks in $e$, where, w.l.o.g, we assume that $p_{1} \leq \ldots \leq p_{n}$. Let $\pi=\sum_{i \in N} p_{i}$ denote the sum of the peaks. We use the following notation: for each $M \in \mathcal{N}$ and each $T \in \mathbb{Z}$, let $\rho(M, T) \in\{0,1\}^{N}$ be a vector whose entries are either 0 or 1 , and such that $\rho(M, T)_{i}=0$ if $i \notin M$ and $\sum_{i \in N} \rho(M, T)_{i}=T$. We distinguish three cases.

Case 1. If $T \leq \pi$. Let us define $\alpha_{k}=\sum_{i \in N} \min \left\{p_{i}, p_{k}\right\}, N_{k}=\left\{i \in N: p_{i} \leq p_{k}\right\}$, and $n_{k}=\left|N_{k}\right|$.

- If $T \in\left[0, \alpha_{1}[\right.$, it is not difficult to check that

$$
\left.\left\{T S^{\sigma}\right\}_{\sigma \in \Sigma_{e}}=\left\{\left(\left\lfloor\frac{T}{n}\right\rfloor, \ldots,\left\lfloor\frac{T}{n}\right\rfloor\right)+\rho\left(N, T-\left\lfloor\frac{T}{n}\right\rfloor \cdot n\right)\right)\right\}_{\rho}
$$

The average of these vectors is equal to $u(e)$.

- If $T \in\left[\alpha_{1}, \alpha_{2}[\right.$,

$$
\left\{T S^{\sigma}\right\}_{\sigma \in \Sigma_{e}}=\left\{\begin{array}{c}
\left(0_{N_{1}},\left\lfloor\frac{T-\sum_{i \in N_{1}} p_{i}}{n-n_{1}}\right\rfloor, \ldots,\left\lfloor\frac{T-\sum_{i \in N_{1}} p_{i}}{n-n_{1}}\right\rfloor\right)+ \\
\rho\left(N-N_{1}, T-\sum_{i \in N_{1}} p_{i}-\left\lfloor\frac{T-\sum_{i \in N_{1}} p_{i}}{n-n_{1}}\right\rfloor\left(n-n_{1}\right)\right)
\end{array}\right\}
$$

The average of these vectors is equal to $u(e)$. 
- In general, if $T \in\left[\alpha_{k}, \alpha_{k+1}[\right.$,

$$
\left\{T S^{\sigma}\right\}_{\sigma \in \Sigma_{e}}=\left\{\begin{array}{c}
\left.\left(0_{N_{k}},\left\lfloor\frac{T-\sum_{i \in N_{k}} p_{i}}{n-n_{k}}\right\rfloor, \ldots, \mid \frac{T-\sum_{i \in N_{k}} p_{i}}{n-n_{k}}\right\rfloor\right)+ \\
\rho\left(N-N_{k}, T-\sum_{i \in N_{k}} p_{i}-\left\lfloor\frac{T-\sum_{i \in N_{k}} p_{i}}{n-n_{k}}\right\rfloor\left(n-n_{k}\right)\right)
\end{array}\right\}
$$

The average of these vectors is equal to $u(e)$.

Case 2. If $T=\pi$, the result is obvious.

Case 3. If $T \geq \pi$. Let us define $\beta_{k}=\pi+\sum_{i \in N} \max \left\{0, p_{k}-p_{i}\right\}, M_{k}=\{i \in N$ : $\left.p_{i} \leq p_{k}\right\}$, and $m_{k}=\left|M_{k}\right|$.

- If $T \in] \beta_{k}, \beta_{k+1}$ ],

$$
\left\{T S^{\sigma}\right\}_{\sigma \in \Sigma_{e}}=\left\{\begin{array}{l}
\left(\left\lfloor\frac{T-\sum_{i \in N-M_{k}} p_{i}}{m_{k}}\right\rfloor, \ldots,\left\lfloor\frac{T-\sum_{i \in N-M_{k}} p_{i}}{m_{k}}\right\rfloor, 0_{-N_{k}}\right)+ \\
\rho\left(M_{k}, T-\sum_{i \in N-M_{k}} p_{i}-\left\lfloor\frac{T-\sum_{i \in N-M_{k}} p_{i}}{m_{k}}\right\rfloor \cdot m_{k}\right)
\end{array}\right\}
$$

The average of these vectors is equal to $u(e)$.

- Finally, if $T>n \cdot p_{n}$

$$
\left.\left\{T S^{\sigma}\right\}_{\sigma \in \Sigma_{e}}=\left\{\left(\left\lfloor\frac{T}{n}\right\rfloor, \ldots,\left\lfloor\frac{T}{n}\right\rfloor\right)+\rho\left(N, T-\left\lfloor\frac{T}{n}\right\rfloor \cdot n\right)\right)\right\}_{\rho}
$$

The average of these vectors is equal to $u(e)$.

We have covered the possible values of $T$.

Open Access This article is distributed under the terms of the Creative Commons Attribution License which permits any use, distribution and reproduction in any medium, provided the original author(s) and source are credited.

\section{References}

Barberà S, Jackson MO, Neme A (1997) Strategy-proof allotment rules. Games Econ Behav 18:1-21 Benassy JP (1982) The economics of market disequilibrium. Academic Press, London Ching S (1994) An alternative characterization of the uniform rule. Soc Choice Welf 11:131-136 Chun Y (1999) Equivalence of axioms for bankruptcy problems. Int J Game Theory 28:511-520 Dagan N (1996) A note on Thomson's characterizations of the uniform rule. J Econ Theory 69:255-261 Herrero C, Martínez R (2008) Balanced allocation methods for claims problems with indivisibilities. Soc Choice Welf 30:603-617

Moulin H (1999) Rationing a commodity along fixed paths. J Econ Theory 84:41-72

Sönmez T (1994) Consistency, monotonicity, and the uniform rule. Econ Lett 46:229-235 
Sprumont Y (1991) The division problem with single-peaked preferences: a characterization of the uniform allocation rule. Econometrica 59:509-519

Thomson W (1994a) Consistent solutions to the problem of fair division when preferences are singlepeaked. J Econ Theory 63:219-245

Thomson W (1994b) Resource-monotonic solutions to the problem of fair division when preferences are single-peaked. Soc Choice Welf 11:205-223

Thomson W (1998a) Consistency and its converse: an introduction. Center for Economics Research, University of Rochester, Rochester, pp 448

Thomson W (1998b) The replacement principle in economies with single-peaked preferences. Soc Choice Welf 15(1):57-66

Young HP (1994) Equity: theory and practice. Princeton University Press, Princeton 